\title{
A review of comet and asteroid statistics
}

\author{
Tom Gehrels
}

Space Science, University of Arizona, Tucson, Arizona 85721, U.S.A.

(Received October 7, 1998; Revised August 12, 1999; Accepted September 15, 1999)

\begin{abstract}
The statistics of Earth-approaching asteroids are first summarized, and an enhanced frequency of objects smaller than 100 meters is noted. Superposed on these random hazards may be a periodic one of new comets due to galactic tides of the Oort Cloud with a period of 26-36 Myr (Rampino, 1998). New asteroids and comets are being found evermore frequently because new telescope-and-detector systems are coming on line. These are intended primarily for the discovery of dangerous objects, but a beginning has been made with the study of statistics of main-belt asteroids. In addition to trans-Neptunian objects, cis-Neptunian "Centaurs" are recognized, which may be a link in the evolution of short-period comets and thereby contribute to the flux of Earth approachers. With the new equipment coming on line, we are beginning to see that the global hazard will be mostly quantified within a few decades. We do see a shortage in astrometric follow up fainter than about the 20th magnitude.
\end{abstract}

\section{Introduction}

Statistics of asteroids were scarcely known until the 1950s when C. J. and I. van Houten made them their lives' dedication in the Yerkes-McDonald Survey (Kuiper et al., 1958) and the Palomar-Leiden surveys (van Houten et al., 1970, 1984, 1989, 1991). The van Houtens did not just do most of the work, but they also took care of bias problems in an exemplary manner (Jedicke, pers. comm., 1999). Before the 1950s also, asteroid magnitudes were not usable for statistics. They could be off by as much as 3 magnitudes; fainter than the 11th magnitude, they tended to be all the same because the Bonner Durchmusterung had been used for calibration and it did not go fainter (Gehrels, 1957, 1970).

In this paper we shall take stock of the present situation in view of a spectacular increase in data that is upon us. An order-of-magnitude increase in finding new asteroids has already happened because of the LINEAR program (Stokes et al., 1998). Other telescopes and detectors are coming on line elsewhere, and this is only the beginning because telescopes with diameters as large as 8 meters are being designed with much wider and deeper coverage (Angel et al., 1999; Armandroff et al., 1999).

In the following Section 2 we shall first overview the statistics of the hazardous asteroids and comets, and in Section 3 see how periodic effects might enter in. In Section 4, the survey programs for dangerous asteroids are listed, while one of them, Spacewatch, is discussed more in detail in Section 5 because it has taken the lead in using the data to analyze comet and asteroid statistics. We shall conclude the paper with a few words in reply to the question that is invariably brought to the fore by the frightening impact statistics: "If a comet or asteroid were found to be headed for the Earth, what can be done about it to avoid the disaster?"

Copy right (C) The Society of Geomagnetism and Earth, Planetary and Space Sciences (SGEPSS); The Seismological Society of Japan; The Volcanological Society of Japan; The Geodetic Society of Japan; The Japanese Society for Planetary Sciences.

\section{Statistics of Earth-Approaching Comets and Asteroids}

Table 1 summarizes our present understanding of the hazard due to comets and asteroids, for which I rounded off the results of Rabinowitz et al. (1994). "Cumulative" in the heading for the second column means that the number of objects is for those equal to or larger than that diameter. The values in the Table are approximate, reflecting the uncertainties of our present knowledge. The most recent determination by Rabinowitz et al. (pers. comm., 1999) of the number in the second column at 1-km diameter is $750 \pm 250$; Bottke, Jedicke and Morbidelli (in preparation, 1999) seem to confirm that number. Other estimates, however, have been as high as 2200, so that this Table still seems appropriate. An improvement may also be made by more narrowly defining the dangerous population. This has been done by Marsden who computes and lists Potentially Hazardous Asteroids (PHA), the ones with absolute magnitude $H<22.0$ (that is larger than about 180 meters in diameter), which may come closer than 0.050 AU from Earth at any time in the foreseeable future. As soon as sufficient data for statistical studies are available we can switch to Marsden's system, but the presently available studies and estimates are still as in Table 1.

The Table shows that for a decline in diameter by a factor of 10 , the number of objects has an increase by a factor of approximately 100 (van Houten et al., 1970, p. 355; Gehrels, 1986, expr. 7). This is because the processes of accretion and collision both depend on the cross section of the target, which is proportional to the square of the radius. Detailed "humps" in the magnitude-frequency relations are seen for instance in the Figure discussed in Section 5 where it is seen that these features are important for studies of origin and collisional evolution.

The fourth column of Table 1 compares the kinetic energy of impact with explosive power expressed in megatons of TNT. It is computed with $1 / 2 m v^{2}$, where $3 \mathrm{gm} / \mathrm{cm}^{3}$ is 
Table 1. Approximate statistics for the hazards due to Earth-approaching Comets and Asteroids.

\begin{tabular}{cccc}
\hline $\begin{array}{c}\text { Diameter } \\
(\mathrm{km})\end{array}$ & $\begin{array}{c}\text { Cumulative } \\
\text { number of } \\
\text { objects }\end{array}$ & $\begin{array}{c}\text { Impact frequency } \\
\text { (once per number } \\
\text { of years) }\end{array}$ & $\begin{array}{r}\text { Impact energy } \\
\text { (equivalent of } \\
\text { megatons TNT) }\end{array}$ \\
\hline 10 & 10 & $10^{8}$ & $10^{7}$ \\
1 & $10^{3}$ & $10^{6}$ & $10^{4}$ \\
0.1 & $10^{5}$ & $10^{4}$ & 10 \\
0.01 & $10^{8}$ & 10 & $10^{-2}$ \\
\hline
\end{tabular}

adopted for the density of mass, $m$. For the velocity, $v$, I took $20 \mathrm{~km} / \mathrm{sec}$. To get an impression of the awful energies of the asteroids, we can compare with the disaster at Hiroshima in 1945, where the atomic bomb had the equivalent of 0.013 megatons of TNT, or $5.4 \times 1020$ ergs.

The only deviation from the above factor of 100 in Table 1 is seen in its bottom line, and it is a striking effect, an additional enhancement at size $0.01 \mathrm{~km}$ by a factor of 10 over the extrapolation from the brighter objects. The effect may actually be as much as a factor of 40 over the above factor of 100; this was a surprising discovery made at the Spacewatch Telescope (Rabinowitz, 1993). Near misses of the Earth by small objects occur frequently (see Scotti et al., 1991); the record stands near 6-m diameter (Guinness Book of Records, 1995, p. 3), and at 105,000 km from the center of the Earth. Various interpretations of the excess at $0.01 \mathrm{~km}$ have been proposed, such as the one in terms of cometary debris (Ceplecha, 1997). Similar excess has, however, been found for main-belt asteroids 20 to 60 meters in diameter (Chapman et al., 1996). Rabinowitz (1997) then found an explanation for the effect on the Earth-approaching asteroids if they originated in the main belt and if there are humps in the size distribution of larger main-belt asteroids. The work of Jedicke and Metcalfe (1998) and of Durda et al. (1998) for the main belt, which will be discussed below in Section 5, established indeed that there are appreciable departures from a power-law distribution.

\section{Studies of Periodicity for Long-Period Comets}

The question is whether Table 1 agrees or not with the theory of a periodicity of some $30 \mathrm{Myr}$ in the major extinctions. Such periodicity was first recognized for the fossils in various geological layerings by Raup and Sepkoski (1984), and for impact craters by Rampino and Stothers (1984) and by Alvarez and Muller (1984). Their analyses were critically reviewed at a conference and refereed for a source book by Smoluchowski et al. (1986), in the Space Science Series of advanced texts published by the University of Arizona Press. An early explanation of that periodicity had been made by postulating a distant brown-dwarf companion of the sun, sweeping in an elliptical orbit every $30 \mathrm{Myr}$ through the outer regions of the solar system, the Oort Cloud of comets (see Oort, 1963). The star had already been named-Nemesis, after the Greek goddess of fate and punishment for extravagant pride-but it was not discovered in telescopic searches, while dynamical studies indicated that its orbit would be un- stable.

More than a decade has gone by and the periodicity analyses are still with us, verified and improved by various investigators. At Smoluchowski's conference, another interpretation had already been discussed, in terms of the undulatory motion of the solar system perpendicular to the galactic plane, which indeed has a half-period of about 30 Myr. The Oort Cloud may thereby be susceptible to tidal bulging as it moves through the gravitational effect of the mass that is concentrated towards the plane of the galaxy. This is now believed to be the cause if indeed there is periodicity in the mass extinctions and impact craterings. It is called the Shiva Effect, which is a better name than Nemesis because Shiva is the Hindu god of regeneration as well as destruction, which is what impacting comets and asteroids accomplish.

Perturbed Oort-Cloud comets would then constitute a periodically active effect superposed on or hidden within the main body of randomness in Table 1 . In and around Table 1 we would see four distinct effects, namely of collisional fragments from the asteroid belt, the remains of burnt-out periodic comets, the enhancement at the bottom of the Table, and the Shiva Effect near its top. The four lines of Table 1 can in any case be thought of as representing successively: mass extinction, global holocaust, regional calamity, and still terrible but localized destruction.

The characteristic of the Shiva Effect, in addition to being periodic, is that the comet diameters are rather large. Mass extinctions could each be due to several comets with core diameters of about $6-12 \mathrm{~km}$; of special interest is the discovery of a multiple impact on Earth by Spray et al. (1998). The conclusions are in agreement with the derivation of impact probability for long-period comets (defined as having periods greater than 200 years) by Marsden and Steel (1994), which also indicates $10^{8}$ for the top line of Table 1 .

The various determinations of the periodicity vary considerably, between 26 and 36 Myr (Rampino, 1998). Napier (1998) derives a periodicity of about 27 Myr. The cratering periodicity tends towards $36 \mathrm{Myr}$ for the comet-derived large craters, while the shorter $26 \mathrm{Myr}$ period is obtained for the extinctions, which may reflect noise from a mixture of periodic and non-periodic events. Further noise may be due to the crossing of the galactic plane being not sharply defined: the precise timing may be affected by varying density of plane material and by individually encountered stars and molecular clouds. Recent impacts have been recognized at approximately 95, 65, 35, and 2-3 Myr ago (Rampino and 
Haggerty, 1996). Even with the last one already 2-3 Myr behind us, its effects may still be with us, still be present in Table 1 (Valtonen et al., 1995).

\section{Survey Programs for Asteroids and Comets}

Table 2 lists the professional telescopes that have been or are still being used to search for dangerous asteroids and comets. Brian Marsden kindly provided the dates for which they have supplied findings of asteroids in general, and of their numbers of Aten, Apollo, Amor discoveries (status of March 18, 1999). At the 0.5-meter Schmidt of the Palomar Observatory, there were teams led by Eugene Shoemaker of the U. S. Geological Survey in Flagstaff, and by Eleanor Helin of the Jet Propulsion Laboratory (JPL) in Pasadena. They used to photograph large areas of the sky with film pairs at half-hour intervals; they discontinued the programs, however, amidst widely shared expectations for switching to charge-coupled devices (CCDs). The 1.2-meter Schmidt on Palomar Mountain is also used for the discovery of Earthapproachers, by various astronomers at various times; the same applies for the 1-meter Schmidt Telescope at the European Southern Observatory in Chile.

The Anglo-Australian program (Steel et al., 1997) at Siding Spring was discontinued by the Australian government at the end of 1996, but asteroid work has been started again with NASA support to Steve Larson and Rob McNaught with a 1-m F/8 reflector and a CCD. The Klet and Ondrejov observatories are both in the Czech Republic. Spacewatch is the name for the program at the University of Arizona, now under the direction of Robert McMillan (1999). China's government has acknowledged the asteroid hazard as their reason for not signing a nuclear proliferation treaty because nuclear weapons may be needed in the mitigation; its Beijing Observatory is discovering Earth-approaching objects (Zhu et al., 1999). The JPL/Hawaii line in Table 2 is for the NearEarth Asteroid Tracking (NEAT; Pravdo et al., 1999). It uses

Table 2. Survey telescopes for asteroids and comets.

\begin{tabular}{lccr}
\hline Identification & Aperture & Discovery years & AAA \\
\hline Palomar Obs. & $0.5 \mathrm{~m}$ & $1973-1995$ & 120 \\
Anglo-Austr. & $1.2 \mathrm{~m}$ & $1974-1996$ & 44 \\
Eur. South. Obs. & $1.0 \mathrm{~m}$ & since 1974 & 13 \\
Klet Obs. & $0.6 \mathrm{~m}$ & since 1978 & 3 \\
Spacewatch & $0.9 \mathrm{~m}$ & since 1984 & 188 \\
Beijing Obs. & $0.6 \mathrm{~m}$ & since 1994 & 5 \\
Ondrejov Obs. & $0.6 \mathrm{~m}$ & since 1994 & 1 \\
JPL/Hawaii & $1.0 \mathrm{~m}$ & since 1995 & 32 \\
Catalina Survey & $0.4 \mathrm{~m}$ & since 1996 & 5 \\
Côte d'Azur & $0.9 \mathrm{~m}$ & since 1996 & 5 \\
Lincoln Lab. & $1.0 \mathrm{~m}$ & since 1996 & 178 \\
Lowell Obs. & $0.6 \mathrm{~m}$ & since 1998 & 7 \\
\hline
\end{tabular}

$\mathrm{AAA}=$ Aten, Apollo, Amor asteroids. an automated CCD system at a telescope on the rim of the Haleakala Crater on Maui, Hawaii; this may soon be done 18 nights per lunation on a 1.2-meter telescope there that has $1.5 \times 1.5$ degree field. NEAT will also be involved with a new wide-angle CCD on the 1.2-m Palomar Schmidt.

The Catalina Sky Survey has recently become highly effective with a CCD camera (Larson et al., 1998). The Observatoire de la Côte d'Azur Survey (ODAS) uses a Schmidt with effective aperture of about 0.9 meter (Scholl et al., 1998). The Lincoln Laboratory uses a telescope, soon two telescopes, in New Mexico with a new type of CCD that it had developed over the years for the U.S. Air Force specially for fast reading out on rapidly moving targets. The software is sophisticated for detecting various objects, also in dense star fields, and it uses the best in computing power; their highly successful program is under the direction of Grant Stokes (Stokes et al., 1998). Ted Bowell manages the Lowell Observatory Near-Earth Object Survey (LONEOS) at Flagstaff, Arizona.

In addition to the list of Table 2 there are other survey systems coming on line, and this also applies to CCD programs of observers who follow the discoveries up with astrometry. At Bisei, Japan, there will be dedicated telescopes of 1-m and 0.5-m aperture for surveying and follow up, respectively ( $\mathrm{S}$. Isobe, pers. comm., 1997). The U.S. Air Force may assign additional surveillance telescopes to this work (S. P. Worden, pers. comm., 1999). We would not be able to consolidate many discoveries without the help of several dozen amateur and professional astronomers who provide the astrometric observations that are essential for good orbits; some of them also discover Earth-approachers.

Not listed in Table 2 are the large telescopes used in discovery of the faint trans-Neptunian objects (TNOs). Jewitt and Luu (1993) discovered the first one as the result of their deliberate and persistent efforts in surveying for these faint objects. They are continuing with spectacular findings (Jewitt et al., 1998), now joined by several astronomers at the largest of telescopes. The number of TNOs stood at about 140 by the middle of 1999. Interpretation of their orbits is reviewed by Malhotra et al. (2000).

All observations are reported to the Minor Planet Center of the International Astronomical Union, located in Cambridge, Massachusetts, where Brian Marsden and Gareth Williams keep track of some $10^{4}$ comets and asteroids.

\section{The Spacewatch Project}

Of the programs in Table 2, Spacewatch is the only one to have the study of comet and asteroid statistics as its primary goal. It was originated for the determination of magnitudefrequency relations for all small-body populations in the solar system; in scientific pursuit it is a CCD extension of the photographic surveys to determine statistics by Kuiper et al. (1958) and by van Houten et al. mentioned at the start of this paper. About 30,000 asteroids per year are presently found at the 0.9-meter Newtonian reflector; results of their statistics are beginning to be published as we saw already for Earth-approaching asteroids in Section 2, and we shall see for others below. Special topics in statistics have been studied for small cometesimals, of which none were found, and for various other objects (Gehrels, 1981, 1986, 1991; 
McMillan, 1999). The observer participates in the discovery of objects by visually scanning the screen all night for faint comets and for trails of objects that move faster than about a degree per day.

For the Earth approachers, Spacewatch searches for smaller as well as for larger ones than the $1.5-\mathrm{km}$ criterion the asteroid community uses for the definition of "global" destruction, the ones menacing with destruction of society and the death of large numbers of people. We are not certain of that definition of "global" destruction; the diameter for that may fall somewhere between 0.6 and $5 \mathrm{~km}$ (Chapman and Morrison, 1994). Nearly all telescopes in Table 2 are aiming at these globally hazardous ones, which are usually brighter than the 20th magnitude. The two Spacewatch telescopes are programmed to search fainter.

The 0.9-meter telescope reaches visual magnitude 21.5 (when the object moves slowly) in routine CCD scanning (sigma 3.7). Four CCDs are on order, $2048 \times 4808$ pixels of 13.5-micron size; the four are buttable on three sides and will be assembled into a mosaic. The 0.9 -meter will be modified from operation at Newtonian F/5 to prime focus $\mathrm{F} / 3$ with a new mirror and a field corrector such that the pixel size is $1 \mathrm{arcsec}$, as before, but now for a field of 2.4 degrees in diameter (McMillan, 1999).

The 1.8-meter telescope (Perry et al., 1998) will be the largest in the world dedicated to surveying, recovery and astrometry of comets and asteroids. It is located near the 0.9-meter in a modern Galvalum-clad building and dome. At $F / 2.7$, it is optimized to yield a field of 0.8 degree in diameter with a CCD of $2048 \times 2048$ pixels, and it should have sufficient aperture and limited pixel size $(1 \operatorname{arcsec})$ to reach visual magnitude 22.5 in routine surveying (McMillan et al., 1998).

There is a trade-off between faintness and area coverage. The LINEAR program covers most of the sky each month when the weather is reasonable, while Spacewatch goes fainter but with much less completion, until the time of delivery of the new detectors, after which there will be more choice in operation. Coordination of the various observing programs of Table 2 will be needed so as to avoid overlap and waste, with discoveries made at one telescope and the observers learning afterwards that the objects had already been discovered elsewhere. A problem that is beginning to be felt already now is the shortage in faint follow up in astrometry, measuring positions at various times, so as to obtain orbits of lasting quality. The discovered objects often get fainter, but there are few amateur or dedicated professional telescopes that can then do such follow up fainter than the 20th magnitude at the time it is needed.

Before being able to make any statistical analysis of the Spacewatch data, Jedicke and his students had to establish the basic calibrations of the system such as its efficiency, or completion, as a function of apparent magnitude. As for statistics of the main-belt asteroids, Jedicke and Metcalfe (1998) analyzed observations of more than 60,000 of them to a limiting magnitude of about $V=21$. This is to be compared with the 1,000 asteroids to $V=16$ by Kuiper et al. (1958) and the 1,800 to $V=19$ by van Houten et al. (1970).

Details of such size distributions have been studied by

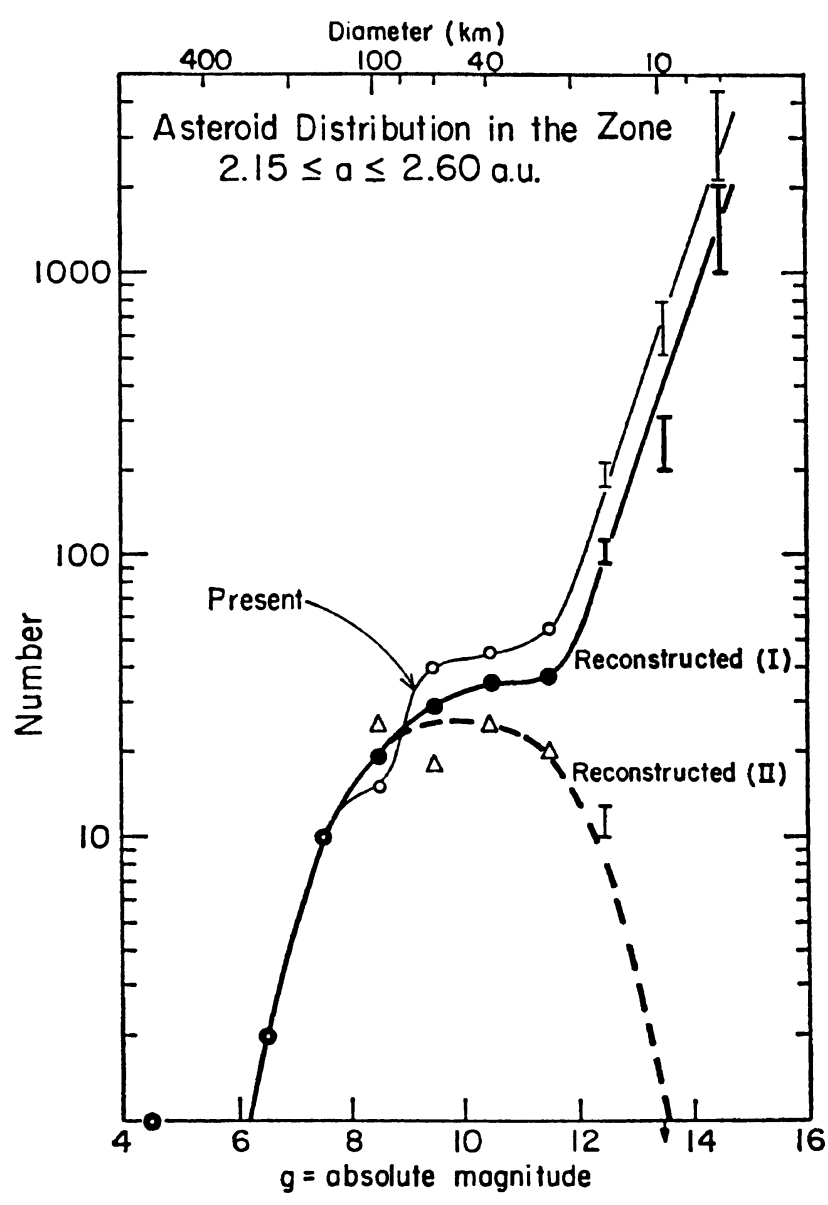

Fig. 1. Reconstruction by Anders (1958) of the magnitude-frequency relation, here called "Present," of Kuiper et al. (1958) for the inner zone of the main asteroid belt; the absolute magnitude $g$ is on a photographic system $(g=H+1)$. When 13 Hirayama families in this zone were reassembled into their parent asteroids, Distribution I resulted. After this operation was repeated, to allow for an equal number of unrecognized or dispersed families, the "fragmental" branch disappeared entirely, leaving Distribution II of approximately Gaussian shape as an approximation to the original distribution.

Anders (1958, see the present Fig. 1), and in modern times by, for instance, Davis et al. (1994), while Durda et al. (1998) used Spacewatch data. Anders had used the observations for the inner zone observed by Kuiper et al. (1958), bright asteroids for which Spacewatch provides few data; the old material is still valuable for such statistical studies. Furthermore, Anders drew detailed conclusions regarding the collisional dispersal that are still interesting. Durda (pers. comm., 1998) notes, however, that "the shape which we have seen evolve, which includes the discontinuity near $20-120 \mathrm{~km}$, is most strongly dependent upon the strength scaling law. The shape of the initial distribution is not very important in the final evolved shape, and in fact in this case most resembled a runaway growth distribution with a collisional tail." The paper by Durda et al. (1998) provides basic information regarding the tensile strength of asteroids of various sizes, which is especially important to know for the mitigation of the asteroid hazard (Section 6). Smaller than about 150 meters in diameter the asteroids must have sufficient intrinsic cohesion, as of rock or metal, instead of their weak gravity, in order to hold together. 
Table 3. Centaurs known to date*.

\begin{tabular}{clccc}
\hline \multicolumn{2}{c}{ Identification } & $\begin{array}{c}\text { Discovery } \\
\text { date }\end{array}$ & $\begin{array}{c}\text { Disc. } \\
\text { magn. }\end{array}$ & Discoverers \\
\hline 2060 & Chiron & 771018 & 19.0 & Kowal \\
5145 & Pholus & 920109 & 16.3 & Rabinowitz \\
7066 & Nessus & 930426 & 20.2 & Rabinowitz \\
& $1994 \mathrm{TA}$ & 941002 & 23.2 & Chen and Jewitt \\
10370 & $1995 \mathrm{DW}_{2}$ & 950227 & 20.9 & Jewitt and Luu \\
8405 & $1995 \mathrm{GO}^{*} 10199$ & 950405 & 20.3 & Scotti \\
& $1995 \mathrm{SN}_{55}$ & 950920 & 22.1 & Gleason \\
& $1997 \mathrm{CU}_{26}$ & 970215 & 17.9 & Scotti \\
& $1998 \mathrm{BU}_{48}$ & 980122 & 21.8 & Danzl \\
& $1998 \mathrm{QM}_{107}$ & 980821 & 23.1 & Whiteley \\
& $1998 \mathrm{SG}_{35}$ & 980919 & 21.3 & Scotti \\
& $1998 \mathrm{TF}_{35}$ & 981012 & 22.4 & Danzl \\
& $1999 \mathrm{HD}_{12}$ & 990417 & 22.9 & Millis and Buie \\
& $1999 \mathrm{JV}_{127}$ & 990510 & 23.8 & Allen and Bernstein \\
\hline
\end{tabular}

*data provided by Marsden (pers. comm., 1999).

Further improvement in the statistical studies will be possible because Spacewatch now observes each field three times per lunation, so that the two intervals are about 6 days, which allows the computation of all orbital elements. Improvements are also underway in constructing a collisional evolution model consistent with the observed asteroid population as well as with the cosmic-ray exposure ages of meteorites and with observed cratering records on Gaspra and other asteroids (R. Greenberg, pers. comm., 1999). However, Durda (pers. comm. to McMillan, 1999) assures us that the diameter at which the transition from tensile strength to gravitational bonding occurs $(\sim 150 \mathrm{~m})$ is insensitive to the changes in the collisional model that may be required to fit the cratering records and cosmic-ray exposure ages.

Beyond the asteroid belt, Spacewatch identified what Kowal and Marsden have named "Centaurs", between the orbits of Jupiter and Neptune. Marsden kindly provided for Table 3 the listing of the 14 Centaurs known on 30 July 1999; the magnitudes are the computed ones for the time of discovery. Nichole Danzl and Arianna Gleason are undergraduate students trained by Jeff Larsen in Spacewatch techniques for finding such faint objects (Larsen, 1998).

Jedicke and Herron (1997) investigated the statistics of the Centaur population. The result is still preliminary as it is essentially based on the only one that met their stringent selection criteria. Nevertheless, their conclusion is that the population may be as large as that in the asteroid belt. Bailey et al. (1992) studied how a chance encounter with Uranus or Saturn might throw them out of the solar system or bring them inward toward the terrestrial planets. In the latter case they may have about $40 \%$ chance of becoming short-period comets of the Jupiter family and evolving into Earth-crossing orbits at a rate of about $1-2 \%$ per million years. When they are in the inner solar system, outgassing and breaking up occurs, which then supply the debris that contributes to the hazard of impacts on the earth.

The cis-Neptunian Centaurs and the trans-Neptunian objects (TNOs) may be closely related and they sometimes can not be distinguished. Marsden (pers. comm., 1999) comments:

Where does one draw the line between a Centaur and a "scattered-disk TNO"? They are the same population. If my proposed identification 1995 $\mathrm{GA}_{7}=1999 \mathrm{KS}_{16}$ is correct (see MPEC 1999036), this object would indeed have its perihelion inside Neptune's orbit, while some of the Centaurs have their aphelia outside Neptune's orbit.

The Spacewatch search is also for low differential velocity, Delta- $V$, candidates for space missions (Lewis et al., 1993), including astrometry and recovery of the ones with such poor orbits that they are considered lost. The best mission candidate presently is $1998 \mathrm{KY}_{26}$, which is an interesting object also because it has the extremely short rotation period of 10.7 minutes. For a diameter of $\sim 30 \mathrm{~m}$, this is a faster rotation than can be held together by its gravity so it must have cohesion of its own (Ostro et al., 1999).

Spacewatch has discovered 13 comets, while Jim Scotti conducts a recovery program that has yielded 60 comets (as of July 1999). The number of Earth-approaching asteroids discovered by Spacewatch with diameters between about $6 \mathrm{~m}$ and $6 \mathrm{~km}$ stands at 197 (also July 1999), and this is increasing at an average of about 22 per year with the present $2048 \times 2048$ pixel CCD system on the 0.9 -meter 
Newtonian reflector. With the new focus and detector system, the discovery rates are expected to increase by an order of magnitude.

There is exciting news also in the statistics of "minor satellites," the nickname for moons of asteroids. The surprise discovery was Dactyl for (243) Ida during the flyby of the Galileo spacecraft. A groundbased search by Merline et al. (1999) paid off with their discovery and orbit determination of S/1998(45)1 as a 13-km satellite in nearly circular orbit of (45) Eugenia. The authors conclude from the searches made by them and by others, mostly made in vain, that asteroidal satellites occur only rarely.

\section{Mitigation}

What if we find an object headed for the earth? This might happen one day; there have been alarms already over $1997 \mathrm{XF}_{11}, 1999 \mathrm{AN}_{10}$, and $1998 \mathrm{OX}_{4}$. Mitigation was overviewed in a source book, as were the discovery and identification of hazardous objects, by 120 experts in 46 refereed chapters (Gehrels, 1994). As editor of that text, I can summarize it as follows. The hazard due to comets and asteroids is the most serious problem humanity faces because it can destroy society globally at once. The energies are millions of times greater than those used at Hiroshima in 1945, while the chance of such a global effect is not negligible. However, with the new telescopes and detectors that are coming on line, and with modern rocketry, it looks likely that the danger of a global holocaust may be nearly eliminated within a few decades.

The method of warding a dangerous object off was seen by means of a "stand-off" explosion of chemical or nuclear warheads that are carried with rockets to near, but not onto, the object to cause a deviation in its orbit. This procedure has been called into question because asteroids may be loose rubble piles (Asphaug et al., 1998; Harris, 1998). Instead, small impulses delivered gradually by thrusters may be safer. There will always be the need for a warning time that is long enough. The technologies of discovery and mitigation seem well within the present state of the art, while they remain lively fields in science and engineering so as to improve the situation steadily into the future.

Acknowledgments. I thank A. Fujiwara for inviting me to write this paper, and B. G. Marsden, R. S. McMillan, D. L. Rabinowitz, M. R. Rampino, D. I. Steel and referees C. R. Chapman and M. Yoshikawa for their helpful comments. The Spacewatch project is supported by NASA, AFOSR, and by private and corporate donors.

\section{References}

Alvarez, W. and R. A. Muller, Evidence from crater ages for periodic impacts on the Earth, Nature, 308, 718-720, 1984.

Anders, E., Fragmentation history of asteroids, Icarus, 4, 399-408, 1958.

Angel, R., M. Lesser, R. Sarlot, and T. Dunham, An 8-meter telescope to image a 3-degree field, 1999 (preprint).

Armandroff, T., G. Bernstein, I. Dell'Antonio, W. Hu, J. Larsen, R. Millis, P. Pinto, A. Tyson, D. Wittman, and D. Zaritsky, Dark matter telescope science drivers, 1999 (preprint).

Asphaug, E., S. J. Ostro, R. S. Hudson, D. J. Scheeres, and W. Benz, Disruption of kilometre-sized asteroids by energetic collisions, Nature, $\mathbf{3 9 3}$, 437-440, 1998.

Bailey, M. E., J. E. Chambers, G. Hahn, J. V. Scotti, and G. Tancredi, Transfer probabilities between Jupiter and Saturn-family orbits: Application to 1992 AD $=5145$, in Observations and Physical Properties of Small Solar System Bodies, edited by J. Surdej and J. C. Gerard, pp. 285-287,
Proc. 30th Liège Int. Astrophys. Colloq., Univ. Liège, Belgium, 1992.

Ceplecha, Z., Influx of large meteoroids onto Earth, Proc. SPIE, 3116, 134 143, 1997.

Chapman, C. R. and D. Morrison, Impacts on the Earth by asteroids and comets: assessing the hazard, Nature, 367, 33-40, 1994.

Chapman, C. R., J. Veverka, M. J. S. Belton, G. Neukum, and D. Morrison, Cratering on Gaspra, Icarus, 120, 231-245, 1996.

Davis, D. R., E. V. Ryan, and P. Farinella, Asteroid collisional evolution: Results from current scaling algorithms, Planet. Space Sci., 42, 599-610, 1994.

Durda, D. D., R. Greenberg, and R. Jedicke, Collisional models and scaling laws: A new interpretation of the shape of the main-belt asteroid size distribution, Icarus, 135, 431-440, 1998.

Gehrels, T., Photometric studies of asteroids. VI. Photographic magnitudes, Astrophys. J., 125, 550-570, 1957

Gehrels, T., Photometry of asteroids, in Surfaces and Interiors of Planets and Satellites, edited by A. Dollfus, pp. 319-376, Academic Press, London, 1970.

Gehrels, T., Faint comet scanning, Icarus, 47, 518-522, 1981.

Gehrels, T., On the feasibility of observing small asteroids with Galileo, Venera, and Comet-Rendezvous-Asteroid flyby missions, Icarus, 66, 288296,1986

Gehrels, T., Scanning with charge-coupled devices, Space Sci. Rev., 58 $347-375,1991$

Gehrels, T., (ed.), Hazards due to Comets and Asteroids, 1300 pp., Univ. Ariz. Press, Tucson, 1994

Harris, A. W., Making and breaking asteroids, Nature, 393, 418-419, 1998.

Jedicke, R. and J. D. Herron, Observational constraints on the Centaur population, Icarus, 127, 494-507, 1997.

Jedicke, R. and T. S. Metcalfe, The orbital and absolute magnitude distributions of main belt asteroids, Icarus, 131, 245-260, 1998.

Jewitt, D. and J. Luu, Discovery of the candidate Kuiper belt object 1992 QB1, Nature, 362, 730-732, 1993.

Jewitt, D., J. Luu, and C. Trujillo, Large Kuiper Belt Objects: The Mauna Kea 8k CCD Survey, Astron. J., 115, 2125, 1998.

Kuiper, G. P., Y. Fujita, T. Gehrels, I. Groeneveld, J. Kent, G. Van Biesbroeck, and C. J. van Houten, Survey of asteroids, Astrophys. J. Suppl., 3, 289-428, 1958.

Larsen, J. A., The Spacewatch survey for bright Kuiper belt objects, Bull. Am. Astron. Soc., 30, 1113, 1998.

Larson, S., J. Brownlee, C. Hergenrother, and T. Spahr, The Catalina sky survey for NEOs, Bull. Am. Astron. Soc., 30, 1037, 1998.

Lewis, J. S., M. S. Matthews, and M. L. Guerrieri, (eds.), Resources of Near-Earth Space, 987 pp., Univ. Ariz. Press, Tucson, 1993.

Malhotra, R., M. J. Duncan, and H. F. Levinson, Dynamics of the Kuiper Belt, in Protostars and Planets IV, edited by V. G. Mannings et al., Univ. Arizona Press, 2000 (in press)

Marsden, B. G. and D. Steel, Warning times and impact probabilities for long-period comets, in Hazards due to Comets and Asteroids, edited by T. Gehrels, pp. 221-239, 1994.

McMillan, R. S., Spacewatch search for material resources near Earth, in Proceedings of the Conference on Space Manufacturing, Space Studies Institute, Princeton, 1999 (in press).

McMillan, R. S., T. H. Bressi, A. S. Descour, T. Gehrels, J. A. Larsen, J. L. Montani, M. L. Perry, M. T. Read, and A. F. Tubbiolo, Progress report on the 1.8-meter Spacewatch telescope, Bull. Am. Astron. Soc., 30, 1114 1998.

Merline, W. J., L. M. Close, C. Dumas, C. R. Chapman, F. Roddier, F. Ménard, D. C. Slater, G. Duvert, C. Shelton, and T. Morgan, Discovery of a moon orbiting the asteroid (45)Eugenia, Nature, 401, 565-568, 1999.

Napier, W. M., NEOs and impacts: The galactic connection, Cel. Mech. Dyn. Astron., 69, 59-75, 1998.

Oort, J. H., Empirical data and the origin of comets, in The Moon, Meteorites and Comets, edited by B. M. Middlehurst and G. P. Kuiper, pp. 665-673, Univ. Chicago Press, 1963.

Ostro, S. J., P. Pravec, L. A. M. Benner, R. S. Hudson, L. Šarounova, M. D. Hicks, D. L. Rabinowitz, J. V. Scotti, D. J. Tholen, M. Wolf, R. F. Jurgens, M. L. Thomas, J. D. Giorgini, P. W. Chodas, D. K. Yeomans, R. Rose, R. Frye, K. D. Rosema, R. Winkler, and M. A. Slade, Radar and optical observations of asteroid 1998 KY26, Science, 285, 557-559, 1999

Perry, M., T. Bressi, R. McMillan, A. Tubbiolo, and L. Barr, The 1.8meter Spacewatch telescope motion control system, in Telescope Control Systems III, Proc. SPIE, 3351, 450-465, 1998.

Pravdo, S. H., D. L. Rabinowitz, E. F. Helin, K. J. Lawrence, R. J. Bambery, 
C. C. Clark, S. L. Groom, S. Levin, J. Lorre, S. B. Shaklan, P. Kervin, J. A. Africano, P. Sydney, and V. Soohoo, The Near-Earth Asteroid Tracking (NEAT) Program: An automated system for telescope control, wide-field imaging, and object detection, Astron. J., 117, 1616-1633, 1999.

Rabinowitz, D. L., The size distribution of the Earth-approaching asteroids, Astrophys. J., 407, 412-427, 1993.

Rabinowitz, D. L., Are main-belt asteroids a sufficient source for the Earthapproaching asteroids? Predicted vs observed orbital distributions, Icarus, 127, 33-54, and 130, 287-295, 1997.

Rabinowitz, D. L., E. Bowell, E. Shoemaker, and K. Muinonen, The population of Earth-crossing asteroids, in Hazards due to Comets and Asteroids, edited by T. Gehrels, pp. 285-312, 1994.

Rampino, M. R., The galactic theory of mass extinctions: an update, $\mathrm{Cel}$. Mech. Dyn. Astron., 69, 49-58, 1998.

Rampino, M. R. and B. M. Haggerty, The "Shiva Hypothesis": Impacts, mass extinctions, and the galaxy, Earth Moon Planets, 71, 441-460, 1996.

Rampino, M. R. and R. B. Stothers, Terrestrial mass extinctions, Cometary impacts and the Sun's motion perpendicular to the galactic plane, Nature, 308, 709-712, 1984.

Raup, D. M. and J. J. Sepkoski, Jr., Periodicity of extinctions in the geologic past, Proc. Natl. Ac. Sc., 81, 801-805, 1984.

Scholl, H., G. Hahn, M. Hoffman, A. Maury, S. Mottola, and ODAS Team, The German-French NEO detection program ODAS, Bull. Am. Astron. Soc., 30, 1037, 1998.

Scotti, J. V., D. L. Rabinowitz, and B. G. Marsden, Near miss by a small asteroid, Nature, 354, 287-289, 1991.

Smoluchowski, R., J. N. Bahcall, and M. S. Matthews, (eds.), The Galaxy and the Solar System, 495 pp., Univ. Ariz. Press, Tucson, 1986.

Spray, J. G., S. P. Kelley, and D. B. Rowley, Evidence for a late Triassic multiple impact event on Earth, Nature, 392, 171-173, 1998.

Steel, D. I., R. H. McNaught, G. J. Garradd, D. J. Asher, and K. S. Russell, AANEAS: A valedictory report, Austral. J. Astron., 7, 67-77, 1997.

Stokes, G. H., H. E. M. Viggh, F. L. Shelly, M. S. Blythe, and J. S Stuart, Results from the Lincoln Near Earth Asteroid Research (LINEAR) Project, Bull. Am. Astron. Soc., 30, 1042, 1998.

Valtonen, M. J., J. Q. Zheng, J. J. Matese, and P. G. Whitman, Near-Earth populations of comets from the Oort Cloud and their impacts with planets, Earth Moon Planets, 71, 219-223, 1995.

van Houten, C. J., I. van Houten-Groeneveld, P. Herget, and T. Gehrels, The Palomar-Leiden survey of faint minor planets, Astron. Astrophys. Suppl., 2, 339-448, 1970.

van Houten, C. J., P. Herget, and B. G. Marsden, The Palomar-Leiden survey of faint minor planets: Conclusion, Icarus, 59, 1-19, 1984.

van Houten-Groeneveld, I., C. J. van Houten, M. Wisse-Schouten, C. Bardwell, and T. Gehrels, The 1977 Palomar-Leiden Trojan survey, Astron. Astrophys., 224, 299-302, 1989.

van Houten, C. J., I. van Houten-Groeneveld, M. Wisse-Schouten, C. Bardwell, D. W. E. Green, and T. Gehrels, The second Palomar-Leiden Trojan survey, Icarus, 19, 326-333, 1991.

Zhu, J., J. Chen, X. Zhou, Z. Zheng, Z. Jiang, Y. Li, L. Deng, B. Zhao, Z. Zheng, H. Wu, S. Xue, and Z. Shang, BAO Schmidt CCD asteroid program: First 4 years' report, Asteroids Comets Meteors, 1999 (submitted).

T. Gehrels (e-mail: tgehrels@u.arizona.edu) 